\title{
What is New in Systemic Lupus Erythematosus: A 2019-2020 Update
}

\author{
Rudrajit Paul ${ }^{1}$, Rathindranath Sarkar ${ }^{2}$
}

\begin{abstract} especially those in the management of SLE.

Bengal Physician Journal (2019): 10.5005/jp-journals-10070-6113

\section{INTRODUCTION}

Systemic lupus erythematosus (SLE) is a multisystem connective tissue disorder with varied manifestations. Our understanding of the disease is evolving with time and hence, the classification criteria, hypotheses of molecular pathophysiology and treatment of the condition are also undergoing paradigm shift very quickly. This article aims to present the current data on some selected aspects of the disease. Clinicians are encouraged to consult current publications for further updates.
\end{abstract}

Scientific understanding of systemic lupus erythematosus (SLE) is progressing at a steady pace. Thus, there has been a paradigm shift in the understanding of the pathogenesis of SLE. As clinical experience of SLE is accumulating, the criteria for diagnosis are also being modified. Treatment of SLE still leaves a lot to be desired but every year, there are new additions and alterations. This article sums up these recent changes,

Keywords: ACR/EULAR, Interferonopathy, Steroids, Systemic lupus erythematosus, TREX-1.

\section{Diagnostic Criteria}

As our understanding of SLE is evolving, the diagnostic criteria are also being modified. The first standard criteria were the 1997 American College of Rheumatology (ACR) criteria, which were replaced by the 2012 Systemic Lupus Erythematosus International Collaborating Clinics (SLICC) criteria. But now, these criteria have also been modified in 2019. It must be remembered that the criteria are mainly developed to be used for clinical trials and translational research. For clinical diagnosis and management, physicians' judgment has no alternative.

\section{The New 2019 ACR/EULAR Criteria}

The new SLE classification criteria are based on a point system. In preliminary studies, these new criteria have been found to have a sensitivity of $96 \%$ and specificity of $94 \%$. Although these criteria have been developed primarily for research, they can also help in clinical practice. All patients suspected of having SLE must have serum antinuclear antibody (ANA) titer 1:80 or above on human Hep- 2 cell lines or similar tests. In addition, there must be a score of 10 or more according to Table 1.

Thus, the clinical criteria have been divided into "domain" with different scores for each manifestation.

\section{Rules for Scoring}

Scores are given for each clinical feature as tabulated above. However, if there are other more plausible explanations for a particular criterion, like oral ulcer, then it is not considered in this
${ }^{1}$ Department of Critical Care Medicine, Institute of Post Graduate Medical Education and Research and Seth Sukhlal Karnani Memorial Hospital, Kolkata, West Bengal, India

${ }^{2}$ Ex-Department of Medicine, Medical College, Kolkata, West Bengal, India Corresponding Author: Rudrajit Paul, Department of Critical Care Medicine, Institute of Post Graduate Medical Education and Research and Seth Sukhlal Karnani Memorial Hospital, Kolkata, West Bengal, India, Phone: +91 9433824341, e-mail: r.paul.medicalcollege@gmail.com

How to cite this article: Paul R, Sarkar R. What is New in Systemic Lupus Erythematosus: A 2019-2020 Update. Bengal Physician Journal 2019;6(2):40-44.

Source of support: Nil

Conflict of interest: None

scoring. Fever has been included as a criterion with two points but alternative etiologies must be ruled out first because fever is a non-specific symptom. Also, only immunological criteria are not enough to diagnose SLE, even if the score is $>10$. At least one clinical criterion must also be present. All the criteria need not be present at the same point of time. Presence of a particular criterion at any point of time in history is enough for scoring, provided it is deemed to be a manifestation of SLE. In a particular domain, as detailed in the chart above, if more than one criterion is present (for example if a patient has both leukopenia and hemolytic anemia), then only the criterion with the highest point score will be considered. Thus, leukopenia has a score of 3 and hemolytic anemia has a score of 4. So, from the hematologic domain, only the score of 4 for hemolytic anemia will be considered.

Initial reports on performance of this criteria have been mixed. For example, Gegenava et al. from Netherlands did a retrospective cohort study using this criterion and found that although the sensitivity was acceptable, the specificity was less than $75 \%$. But Aringer et al. did a multicenter study and reported that the sensitivity and specificity of this new criteria are quite good. Thus, these are early times to draw any conclusion and only further studies in future will clarify the issue.

() The Author(s). 2019 Open Access This article is distributed under the terms of the Creative Commons Attribution 4.0 International License (https://creativecommons. org/licenses/by-nc/4.0/), which permits unrestricted use, distribution, and non-commercial reproduction in any medium, provided you give appropriate credit to the original author(s) and the source, provide a link to the Creative Commons license, and indicate if changes were made. The Creative Commons Public Domain Dedication waiver (http://creativecommons.org/publicdomain/zero/1.0/) applies to the data made available in this article, unless otherwise stated. 
Table 1: Points under clinical and immunological criteria

\begin{tabular}{|c|c|c|c|c|c|}
\hline \multicolumn{2}{|l|}{ Clinical criteria } & \multirow{2}{*}{$\frac{\text { Points }}{2}$} & \multicolumn{2}{|l|}{ Immunological criteria } & \multirow{2}{*}{$\frac{\text { Points }}{2}$} \\
\hline Constitutional domain & Fever & & $\begin{array}{l}\text { Anti-phospholipid } \\
\text { antibody domain }\end{array}$ & $\begin{array}{l}\text { Anti-cardiolipin IgG } \\
>40 \mathrm{GPL} \text { or anti- } \beta 2 \mathrm{GPI} \\
>40 \text { units or lupus } \\
\text { anticoagulant positive }\end{array}$ & \\
\hline \multirow[t]{4}{*}{ Cutaneous domain } & Non-scarring alopecia & 2 & \multirow{2}{*}{$\begin{array}{l}\text { Complement protein } \\
\text { domain }\end{array}$} & Low C3 or low C4 & 3 \\
\hline & Oral ulcer & 2 & & Low C3 and low C4 & 4 \\
\hline & Acute cutaneous lupus & 6 & \multirow{2}{*}{$\begin{array}{l}\text { Highly specific } \\
\text { antibodies domain }\end{array}$} & Anti-dsDNA & 6 \\
\hline & $\begin{array}{l}\text { Subacute cutaneous or discoid } \\
\text { lupus }\end{array}$ & 4 & & Anti-Sm & 6 \\
\hline Arthritis domain & $\begin{array}{l}\text { Synovitis in at least two joints or } \\
\text { tenderness in at least two joints, } \\
\text { along with at least } 30 \text { minutes of } \\
\text { morning stiffness }\end{array}$ & 6 & & & \\
\hline \multirow[t]{3}{*}{ Neurologic domain } & Delirium & 2 & & & \\
\hline & Psychosis & 3 & & & \\
\hline & Seizure & 5 & & & \\
\hline \multirow[t]{2}{*}{ Serositis domain } & Pleural or pericardial effusion & 5 & & & \\
\hline & Acute pericarditis & 6 & & & \\
\hline \multirow[t]{3}{*}{ Hematologic domain } & Leukopenia & 3 & & & \\
\hline & Thrombocytopenia & 4 & & & \\
\hline & Autoimmune hemolysis & 4 & & & \\
\hline \multirow[t]{3}{*}{ Renal domain } & Proteinuria $>0.5 \mathrm{~g} / 24$ hours & 4 & & & \\
\hline & Class II or V lupus nephritis & 8 & & & \\
\hline & Class III or IV lupus nephritis & 10 & & & \\
\hline
\end{tabular}

\section{Treatment}

New treatment protocol: 2019 EULAR recommendations. ${ }^{1}$

The following paragraphs give a summary of the key points in this recommendation:

The main goals of treatment of SLE are remission of disease activity and prevention of flares. For this, various therapies may need to be adjusted and new therapies may be added at different points in the course of the disease.

Hydroxychloroquine should be started in all SLE patients at doses not exceeding $5 \mathrm{mg} / \mathrm{kg} /$ day of actual body weight. Along with it, oral steroids may be needed in significant organ involvement. The use of glucocorticoids depends on the severity of organ involvement. For severe manifestations like renal failure, higher oral steroid doses will be required long-term. This raises the risk of side effects.

An alternative protocol may also be followed. Pulse methylprednisolone can be used at the beginning of treatment followed by oral steroids. This can help reduce the oral dose of steroids later. This will help reduce the various steroid-related side effects in the long run. Oral steroids should be quickly minimized to $<7.5 \mathrm{mg} /$ day and withdrawn as early as possible. For patients not controlled on HCQ and steroids or in whom steroid doses can't be tapered, other immunomodulatory therapies like mycophenolate, methotrexate or azathioprine should be added quickly, instead of persisting with the high dose oral steroid. However, if there is organ threatening disease like neurolupus, such immunomodulators can be added from the very beginning. Cyclophosphamide is only used in severe disease (like renal flare) or as rescue therapy.

Biologic agents useful in SLE include belimumab and rituximab.

There are certain systemic involvements in which other special drugs can be used selectively, in addition to the first line drugs mentioned above. This includes retinoids and dapsone for skin diseases, IVIG or cyclosporine for hematological manifestations, antiplatelet agents for APLA or cardiovascular diseases. The use of calcineurin inhibitors for renal disease is also practiced in certain centers but it has not been adequately studied in all populations.

\section{Steroids in Systemic Lupus Erythematosus}

The use of steroids in SLE is a topic of debate. In, Ruiz-Irastorza et al. found that use of high dose steroids in the first month after the diagnosis of SLE was a predictor of subsequent high steroid burden. ${ }^{2}$ In this study, "high dose" has been defined as $>30 \mathrm{mg} /$ day. Prolonged high dose steroid use is associated with complications like diabetes, cataract and osteonecrosis. Thus, it was proposed that if initial steroid doses were low, then subsequent cumulative dose will also be low. But there is a counter-argument. This states that initial high steroid dose is used in patients with severe disease at onset, who need to continue intensive therapy subsequently. Thus high steroid dose is a marker of high disease activity and not something which can be changed at will. But such argument is only partially valid because subsequent high steroid dose was independent of SLE disease activity index (SLEDAI) score at baseline and use of steroid sparing agents led to the same state of remission as high dose steroids. Thus, it seems there is indeed a window of opportunity for reducing steroid dose in many SLE patients and every effort should be made to reduce oral steroids to $<7.5 \mathrm{mg} /$ day as early as possible.

This concept led to formulation of the treat to target approach for SLE. ${ }^{3}$ As SLE patients are living longer, the side effects of prolonged treatment are becoming more visible. Hence, steroid dose reduction is now an important issue. Along with achieving SLEDAI-2K scores and preventing flares, 
one of the targets in this approach is to achieve a prednisolone daily dose $\leq 7.5 \mathrm{mg} /$ day. But in a recent study by Piga et al., it was found that the most common reason for failing to achieve this target was inability to reduce the steroid dose. ${ }^{4}$ Renal involvement at onset was the main predictor of failure to achieve targets.

Thus, the current expert opinion is to start with low dose $(0.5 \mathrm{mg} / \mathrm{kg})$ of oral prednisolone and taper it as soon as possible, with steroid sparing agents if needed.

\section{Some Newer Experimental Therapies}

Laquinimod, a small molecule NF-KB inhibitor, which inhibits release of proinflammatory cytokines, has been tried in murine lupus nephritis models. In a small phase 2 human trial, it was found to improve kidney function. But it is still nowhere near clinical application.

Similarly, another pathway which has been targeted in lupus nephritis treatment is proteasome pathway. Proteasome inhibitors have been successfully used to treat murine lupus.

There was a trial called ACCESS trial which tested the efficacy of abatacept in inducing remission in lupus nephritis and preventing subsequent flares. The study was not adequately powered to test the efficacy of abatacept in maintaining remission but the initial results have been encouraging.

\section{Key Molecular Pathways}

Systemic lupus erythematosus is an autoimmune disease caused by various pro-inflammatory pathways. One of the pathways, which has gained prominence in recent times is IFN type $1 .{ }^{5}$ There is a new name for this pathogenic mechanism: interferonopathy. When the type I IFN pathway is activated, it gives rise to interferon gene signatures (IFNGS), which is found in autoimmune diseases like SLE.

In some studies, more than $80 \%$ of SLE patients has been found to have the IFNGS. ${ }^{6}$ However, it is still not known whether this IFNGS correlates with disease phenotype. There are other markers like plasmablast and neutrophil signatures, which may correlate with disease activity. ${ }^{5}$ The exact stimulus for IFN1 production in SLE is not known. But based on microbiological studies, cytosolic and endosomal nucleic acid sensors are probably the key initiator of this pathway in SLE. ${ }^{5}$ Furthermore, there may also be genetic predisposition to IFN-1 production, and thus genesis of SLE. STAT4 risk allele is one such potential genetic factor. ${ }^{7}$ Epigenetic mechanisms may also be responsible. For example, differential methylation of type I IFN responsive genes may be a factor in the pathogenesis of SLE. ${ }^{5}$

There are many other molecular pathways which are important in the pathogenesis of SLE. For example, there is mitochondrial dysfunction of the T cells in SLE. There is mitochondrial hyperpolarization and decreased synthesis of ATP. There is also decreased IL-2 production in CD4 cells.

\section{Genetic Factors}

A new paradigm in the pathogenesis of lupus is monogenic lupus. In the recent years, loads of new data have emerged on the genetic basis of SLE. More than 80 new loci have been identified to be associated with SLE, both polygenic and monogenic. ${ }^{8}$ Table 2 mentions some of the genes associated with monogenic lupus or lupus like states. ${ }^{8}$

This list is not exhaustive and other similar candidate genes may be found in standard publications. Here, the authors will elaborate a bit on Aicardi-Goutières syndrome (AGS).
Table 2: Genes associated with monogenic lupus

\begin{tabular}{|c|c|c|}
\hline Gene & Function & Syndrome \\
\hline ACP5 & Acid phosphatase & SPENCD SLE \\
\hline C1QA & $\begin{array}{l}\text { Complement C1q A } \\
\text { chain }\end{array}$ & Complement deficiency SLE \\
\hline DNASE1L3 & Deoxyribonuclease & Classical SLE \\
\hline ISG15 & Ubiquitin like modifier & Aicardi-Goutières syndrome \\
\hline PSMB8 & Proteasome subunit & CANDLE \\
\hline RNASEH2C & Ribonuclease subunit & Aicardi-Goutières syndrome \\
\hline TMEM173 & Transmembrane protein & $\begin{array}{l}\text { STING-associated vasculo- } \\
\text { pathy with onset in infancy }\end{array}$ \\
\hline
\end{tabular}

AGS is a syndrome of monogenic lupus. It is mainly a neurodevelopmental disorder with some features overlapping with SLE. There are multiple clinical features of this syndrome. ${ }^{8}$ It starts in infancy with features like congenital viral infection. There may be encephalopathy and on MRI, white matter changes may be found. There may be multiple autoantibodies: against nuclear factors, gliadin or basement membrane components. IFNGS is also found. There is another variant of AGS which manifests later in life and it has milder features.

There are many mutations which may be responsible for AGS. TREX1 mutation is a common feature. This is a primer exonuclease, involved in nucleic acid degradation pathways. ${ }^{8}$ This mutation is responsible for autoimmune features like leukopenia, oral ulcer and arthritis. These patients usually present in the neonatal period. Neurological impairment is usually severe.

Monogenic lupus is usually inherited as autosomal recessive disease (like TREX 1 mutation). However, some like familial chilblain lupus (FCL) are inherited in an autosomal dominant manner. ${ }^{9}$ Usually, the mutations causing lupus are loss-of-function mutations. But sometimes gain-of-function mutations like IFIH1 gene mutation can cause lupus like features with Jaccoud's arthropathy.

Complement factor deficiency due to mutation is another major pathway for the pathogenesis of lupus. C1q deficiency is associated with SLE like features $90 \%$ of the time with features like photosensitive skin rash, nephritis or arthritis. ${ }^{10}$ These patients present in childhood. Plasma transfusion can sometimes abate the symptoms. Bone marrow transplant is said to be curative. The most common complement factor deficiency in the European population is C2 deficiency. In around $10 \%$ of cases of congenital C2 deficiency, SLE like features may develop. ${ }^{11}$ These patients are usually similar to the general SLE population in terms of age of onset and clinical features. In most cases, they are managed like normal SLE patients and C2 levels are not checked. It does not make any difference in the prognosis. Deficiency of C4 is also associated with SLE-like features.

Rarely, genes causing Noonan syndrome like PTPN11 and SHOC2 are also associated with lupus like features. ${ }^{8}$

\section{ConCLUSION}

With improved understanding of the various aspects of SLE, concepts about diagnosis and management are undergoing a paradigm shift. Clinicians should be aware of the nuances of various evolving concepts.

\section{References}

1. Fanouriakis A, Kostopoulou M, Alunno A, et al. 2019 update of the EULAR recommendations for the management of systemic lupus 
erythematosus. Ann Rheum Dis 2019;78(6):736-745. DOI: 10.1136/ annrheumdis-2019-215089.

2. Ruiz-Irastorza G, Garcia M, Espinosa G, et al. First month prednisone dose predicts prednisone burden during the following 11 months: an observational study from the RELES cohort. Lupus Sci Med 2016;3(1):e000153. DOI: 10.1136/lupus-2016000153.

3. van Vollenhoven RF, Mosca M, Bertsias G, et al. Treat-to-target in systemic lupus erythematosus: recommendations from an international task force. Ann Rheum Dis 2014;73(6):958-967. DOI: 10.1136/annrheumdis-2013-205139.

4. Piga M, Floris A, Cappellazzo G, et al. Failure to achieve lupus low disease activity state (LLDAS) six months after diagnosis is associated with early damage accrual in Caucasian patients with systemic lupus erythematosus. Arthritis Res Ther 2017;19(1):247. DOI: 10.1186/ s13075-017-1451-5.

5. Crow MK, Ronnblom L. Type I interferons in host defense and inflammatory diseases. Lupus Sci Med 2019;6(1):e000336. DOI: 10.1136/lupus-2019-000336.
6. Banchereau R, Hong S, Cantarel B, etal. Personalized immunomonitoring uncovers molecular networks that stratify lupus patients. Cell 2016;165(3):551-565. DOI: 10.1016/j.cell.2016.03.008.

7. Hagberg $\mathrm{N}$, Joelsson $\mathrm{M}$, Leonard $\mathrm{D}$, et al. The STAT4 SLE risk allele rs7574865[T] is associated with increased IL-12-induced IFN- $\gamma$ production in T cells from patients with SLE. Ann Rheum Dis 2018;77(7): 1070-1077. DOI: 10.1136/annrheumdis-2017-212794.

8. Alerin JM, Ortiz-Fernández L, Sawalha AH. Monogenic lupus: a developing paradigm of disease. Front Immunol 2018;9:2496. DOI: 10.3389/fimmu.2018.02496.

9. Rice Gl, Rodero MP, Crow YJ. Human disease phenotypes associated with mutations in TREX1. J Clin Immunol 2015;35(3):235-243. DOI: 10.1007/s10875-015-0147-3.

10. Hagberg N, Rönnblom L. Systemic lupus erythematosus-a disease with a dysregulated type I interferon system. Scand J Immunol 2015;82(3):199-207. DOI: 10.1111/sji.12330.

11. Macedo $A C L$, Isaac L. Systemic lupus erythematosus and deficiencies of early components of the complement classical pathway. Front Immunol 2016;7:55. DOI: 10.3389/fimmu.2016.00055. 


\section{MCQ}

1. What is the minimum score required for diagnosis of SLE in the new 2019 ACR/EULAR criteria?
1. 10
2. 12
3. 9
4. 16

2. Which of the following is not an interferonopathy?
1. SLE
2. SAVI
3. Proteasome associated autoinflammatory syndrome
4. Amyloidosis

3. Which if the following is not included in the recent "treat to target" in SLE?

1. Aim for the lowest glucocorticoid dose

2. Inclusion of anti-malarials in the treatment

3. At least 1 year of immunosuppressive maintenance for lupus nephritis

4. Prevention of flares

4. What is the correct mechanism of action of Belimumab?

1. Inhibition of T cells

2. Reduction in TNF action

3. Inhibition of B-cell activating factor

4. Epigenetic modification

5. Which of the following mutations is responsible for Aicardi-Goutières syndrome?
1. ATP7B
2. TREX1
3. SCN5A
4. PTPN11

\section{Answers:}

$\begin{array}{lllll}\text { 1. A 2.D } & \text { 3.C } & \text { 4.C } & \text { 5.B. }\end{array}$ 